A N N A L E S Annales de Bretagne et des Pays de l'Ouest

\title{
Du comptoir à la toge
}

Antoine Anthoine : négoce, familles et pouvoirs en Provence au XVIIIe siècle

\section{Gilbert Buti}

\section{OpenEdition}

\section{Journals}

Édition électronique

URL : http://journals.openedition.org/abpo/1057

DOI : $10.4000 /$ abpo. 1057

ISBN : 978-2-7535-1500-0

ISSN : 2108-6443

Éditeur

Presses universitaires de Rennes

Édition imprimée

Date de publication : 20 décembre 2005

Pagination : 201-215

ISBN : 978-2-7535-0243-7

ISSN : 0399-0826

Référence électronique

Gilbert Buti, « Du comptoir à la toge », Annales de Bretagne et des Pays de l'Ouest [En ligne], 112-4 | 2005 mis en ligne le 20 décembre 2007, consulté le 02 mai 2019. URL : http://journals.openedition.org/ abpo/1057 ; DOI : 10.4000/abpo.1057 


\title{
Du comptoir à la toge
}

\section{Antoine Anthoine : négoce, familles et pouvoirs en Provence au XVIII ${ }^{\mathrm{e}}$ siècle}

\author{
Gilbert BuTI \\ Maître de conférences en histoire moderne \\ UMR Telemme - Université de Provence (Aix-en-Provence)
}

Écrite par Jean-Louis Antiboul, ancien capitaine, marchand et magistrat de Saint-Tropez, la longue dédicace au roman Abel et Marie ne souligne-elle pas dans une certaine mesure les liens unissant ces deux composantes?

" Il y a plus de quarante ans que je vous ai laissés, non par lassitude et dégoût du noble état de marin, mais forcé par les suffrages de mes concitoyens et les désirs de mes parents qui me firent quitter le compas, la carte, les instruments nautiques et le comptoir pour endosser la toge. Depuis notre séparation, quoique uniquement occupé de mon nouvel état auquel je ne comprenais rien et que je voulais apprendre, je n'ai pourtant pas cessé de vous avoir présents à ma pensée.

[....] Me considérant toujours comme un marin et rêvant sans cesse à la gloire et à la prospérité de notre marine je n'ai pas voulu faire le voyage de l'autre monde sans lui faire mes adieux [...]

Antiboul, ancien magistrat et vieux marin ${ }^{1}$."

Effet de style ou véritable émotion chez ce vieux marin? Quelles sont, pour lui, la part du choix et la place de la contrainte dans l'endossement de la toge? Cette confession d'un enfant du Siècle des Lumières n'est-elle pas quelque peu étonnante lorsqu'on connaît le passé marchand des Antiboul, leurs stratégies et leurs efforts pour atteindre les plus hautes marches des pouvoirs locaux, régionaux et nationaux? L'itinéraire de l'un d'entre eux, Charles-Louis, frère de Jean-Louis, magistrat conventionnel guillotiné en 1794 pour s'être opposé comme d'autres Girondins à l'exécution de Louis XVI, en serait une brillante illustration.

Ces stratégies se croisent chez d'autres représentants du monde de la mer et de la marchandise dans la société littorale provençale au XVIII ${ }^{\mathrm{e}}$ siècle, aussi bien à Marseille, première place marchande, que dans les ports secondaires qui composent son complexe portuaire.

1. Arch. dép. du Var, 18 J 1812. 
Ainsi, à l'entrée du cimetière Saint-Pierre de Marseille, nous pouvons lire sur la tombe du négociant Antoine Anthoine l'inscription suivante :

\author{
" Négociant utile à sa patrie, \\ chargé de missions honorables \\ en Russie et en Pologne, \\ il réussit à ouvrir et à assurer \\ le commerce de la mer Noire \\ avec le Midi de la France. \\ Maire de Marseille, \\ il mérita, pendant huit ans, \\ dans l'exercice de ses fonctions, \\ l'estime de ses concitoyens ${ }^{2}$."
}

Quelles places les familles, les groupes ou les atomes sociaux ${ }^{3}$, ont-ils occupées dans cette trajectoire individuelle où se trouvent également associés la mer, le comptoir et la toge? Certes, la thématique ne prétend pas à la nouveauté. Des travaux anciens et d'autres plus récents ont en effet tracé de multiples voies dans cette direction ${ }^{4}$. Toutefois, elle mérite d'être sinon revisitée tout au moins empruntée et prolongée pour décrypter certains mécanismes et approcher les logiques de fonctionnement.

L'angle d'attaque demeure pourtant délicat à définir. Nous nous heurtons en effet à un choix en rien original : suivre un itinéraire individuel ou opter pour un tableau général. Une trajectoire marchande, en l'occurrence celle du négociant marseillais Antoine Anthoine, restituée au fil d'une microanalyse, reste unique par définition et ne prétend pas à l'exemplarité. Ce négociant, qui a joué un rôle singulier dans l'histoire du commerce de Marseille, a participé au XVIII ${ }^{\mathrm{e}}$ siècle à la puissante dilatation de l'espace commercial qui a hissé la cité au niveau de port mondial. Ses initiatives économiques et ses innovations commerciales l'ont placé parmi les " pionniers " du monde du négoce, avant de le conduire sur les plus hautes marches du pouvoir municipal. Est-ce là un des objectifs majeurs de ces hommes? Atteindre, seul ou en tant que représentant d'un groupe, les pouvoirs pour les mettre à la disposition de leurs ambitions économiques ou bien pour renoncer à la marchandise et se consacrer à d'autres horizons?

Déchiffrer les linéaments de l'itinéraire d'un acteur économique au travers le microcosme reconstitué, de sa formation au comptoir aux hautes charges de la ville, conduit inévitablement à croiser ces interrogations. Bien que non représentatif de l'ensemble du groupe des négociants, le parcours d'Antoine Anthoine permet d'apercevoir les connexions et les logiques qui peuvent exister entre économie et pouvoir. Une mince pellicule ne peutelle pas témoigner sur l'épaisseur du tout?

2. Notice nécrologique de A. I. Anthoine dans LaUTARD, Histoire de l'Académie de Marseille, Marseille, 1829.

3. DellLLe, Gérard, Le Maire et le prieur. Pouvoir central et pouvoir local en Méditerranée occidentale (XV ${ }^{e}$-XVIII siècle), Paris, 2002.

4. Pour une mise en perspective on se reportera à la contribution de Jean-Philippe Priotti. 


\section{Le comptoir à défaut de la toge (1767-1771)}

\section{La famille}

Aîné d'une famille de sept enfants, Antoine Ignace Anthoine est né en 1749, dans les Hautes-Alpes. Son père, avocat au Parlement, occupait la charge de lieutenant général de police de la ville d'Embrun en Dauphiné, ville où, comme nombre d'enfants de ce groupe de petits notables, Antoine suivit des études au collège des Jésuites ${ }^{5}$. Cependant, au terme de cette formation, il ne put prétendre à la succession de son père étant donné le coût trop élevé d'une charge dans la magistrature et les possibilités alors limitées offertes à Embrun tout comme à Gap ou Briançon.

Le commerce apparut comme une orientation possible pour le jeune garçon dans la mesure où parmi les alliés et les amis de la famille Anthoine, nombreux exerçaient déjà, à des niveaux très divers, le métier de "marchand ". D'aucuns étaient installés sur place mais la plupart hors du Dauphiné. Ne pouvant donc revêtir la toge, Antoine fut dirigé vers le comptoir. La formation juridique, parfois considérée comme un tremplin pour une future carrière politique, après un passage au comptoir, ne correspond pas à la trajectoire esquissée par la formation du jeune Anthoine.

\section{L'apprentissage au comptoir}

Vers 1767, Antoine Anthoine, âgé de 18 ans, fut envoyé à Marseille pour faire son apprentissage. La ville portuaire avait acquis au cours du $\mathrm{XVIII}^{\mathrm{e}}$ siècle une stature internationale. Son rayonnement débordait la Méditerranée familière, des proches péninsules au monde arabo-musulman, et mordait sur les lointains espaces océaniques, atlantique et indien. La dilatation de l'espace commercial s'était accompagnée de l'émergence d'un puissant groupe de négociants, répartis en ce milieu de siècle, au sein d'environ cinq cents maisons d'importance très inégale ${ }^{6}$.

Antoine fut placé chez l'une d'entre elles, de moyenne envergure, dirigée par Alexis Rostand, marchand drapier originaire d'Orgon. Ce choix paraît avoir été guidé avant tout par des liens de parenté renforcés par le réseau protestant. En effet, la grand-mère maternelle du jeune Antoine, Thérèse Lions, épouse d'Antoine Rous de la Mazelière et fille d'Isaac Lions, marchand et protestant, était apparentée à l'épouse d'Alexis Rostand, à savoir Marguerite Lions, de Toulon. Cette relation familiale semble avoir été à la base de l'installation d'Antoine Anthoine chez les Rostand ${ }^{7}$.

5. MoretTI, Xavier, « Antoine Ignace Anthoine, baron de Saint-Joseph », Provincia, t. XIX, 1939.

6. CARRIERE, Charles, Négociants marseillais au XVIII siècle. Contribution à l'étude des économies maritimes, 2 volumes, Marseille, 1973.

7. DioQuE, Georges, Un haut-alpin à Marseille. Le baron Anthoine (1749-1826). Du grand négoce à la mairie, Gap, 1991, p. 46-47. 
Après quelques mois d'apprentissage, le marchand-drapier parvint à introduire Antoine dans la maison de commerce des Seimandy, grands négociants de Marseille, protestants venus de Bédarieux, en Languedoc. Par cette décision, Antoine se trouvait désormais employé par une des plus puissantes maisons de la place. Les liens économiques rendent compte avant tout de ce choix dans la mesure où A. Rostand était en étroites relations d'affaires avec les Seimandy pour le commerce des draps entre le Languedoc et le Levant.

Antoine Anthoine fut commis au comptoir des Seimandy, ensemble regroupant à la fois magasins, remises et ateliers. Il reçut chez eux une classique formation négociante ${ }^{8}$ faite de copies de lettres, de classement de la correspondance, de connaissements, de lettres de voiture, de tenue de la comptabilité, de rédaction de factures, de lettres de change, de polices d'assurances, sans omettre les lectures attentives du Parfait négociant ${ }^{9}$ et du Dictionnaire universel du commerce ${ }^{10}$. Cette première formation semble avoir donné entière satisfaction aux Seimandy dans la mesure où en 1771, soit après deux ans d'apprentissage au comptoir marseillais, ils déléguèrent Antoine Anthoine à Constantinople pour y prendre la direction d'une de leurs commandites. Au vrai, cette pratique était amplement répandue sur la place marseillaise : la fonction de régisseur à laquelle accédait Antoine Anthoine se trouvait souvent ouverte aux fils de négociants pour parfaire leur formation ${ }^{11}$.

Ainsi, dans cette première étape de la formation d'Anthoine se combinèrent de classiques éléments sans que l'initiative ait été totalement sienne : les liens familiaux, les réseaux de parentèles, les relations d'affaires d'autant plus efficaces que mettant en jeu de puissants partenaires.

\section{Initiative économique et soutien des pouvoirs (1771-1785)}

\section{Acteur économique à Constantinople : 1771-1781}

Répondant aux souhaits de Jacques Seimandy, Antoine Anthoine rejoignit, à l'âge de vingt-deux ans, la nation française de Constantinople grosse d'environ deux cent cinquante personnes et établie dans le quartier de Péra. Rapidement, par ambition, par tempérament et bénéficiant du réseau de la puissante maison marseillaise, il fut élu " député " de la nation française. Cette fonction recouvrait un rôle économique, politique et diplomatique ponctué de régulières rencontres avec l'ambassadeur, le comte de SaintPriest qui venait de succéder, en 1768, au comte de Vergennes.

Par ce choix, il s'agissait pour le jeune Antoine de représenter les autres marchands français de la place tout en maintenant des liens privilégiés avec

8. Angiolini, Franco, Roche, Daniel (dir.), Cultures et formations négociantes dans l'Europe moderne, Paris, 1995.

9. SAVARY, Jacques, Le Parfait négociant, Paris, $1^{\text {re }}$ édition, 1675.

10. Savary des Bruslons, Dictionnaire universel du commerce, Paris, $1^{\text {re }}$ édition, 1723-1730.

11. PARIS, Robert, Histoire du commerce de Marseille, t. V, Le Levant, Paris, 1957. 
le majeur ou commanditaire. Il devait connaître les partenaires commerciaux présents à Constantinople et à Smyrne, apprendre quelques rudiments (poids, mesures, monnaies, langues), nouer des liens avec les drogmans (personnages-clés dans nombre de contacts) ${ }^{12}$, mais aussi informer au mieux les Seimandy, réfléchir et proposer d'éventuelles opérations commerciales; ainsi Antoine songea-t-il à l'établissement de nouvelles liaisons avec la Perse et l'Inde ainsi qu'avec la Russie méridionale.

L'année 1774 marqua, à plus d'un titre, la carrière du jeune régisseur. Le " krach " des courtiers à Marseille, entraînant la faillite des Seimandy, lui imposa indirectement une certaine indépendance qui le conduisit à travailler avec d'autres négociants marseillais comme les Greling, Peschier et Bouillon ${ }^{13}$. Par ailleurs, à la suite de sa défaite contre la Russie, l'Empire ottoman dut accorder aux vainqueurs la liberté de circuler en mer Noire (Khaïnardji, 1774) et l'ouverture des Détroits (Aïnali Kavac, 1779). Les premières réalisations françaises vers la Crimée remontaient au début du siècle mais l'impossibilité de faire ouvrir la mer Noire au pavillon français avait limité toute entreprise dans cette direction. Le succès russe permit de nouvelles perspectives. En effet, Anthoine entendit mettre à profit la nouvelle donne commerciale pour utiliser la mer Noire, et non plus la Baltique via l'Angleterre et la Hollande, dans l'approvisionnement de Marseille et Toulon en produits nordiques et russes, comme les grains, le suif, le goudron et les bois. Dans ce but, il rédigea un Mémoire qu'il soumit aux autorités locales, sachant pertinemment que l'ambassadeur Saint-Priest poussait les Marseillais dans cette voie. Jeu subtil où se mêlaient visée commerciale, projet politique et ambition personnelle. Anthoine parvint donc sans difficulté à obtenir l'approbation de l'ambassadeur français, tout disposé à l'être, mais aussi de l'ambassadeur russe Stachiev qui se trouvait dans de semblables dispositions ${ }^{14}$.

Assurément, l'interaction apparaît ici évidente entre les objectifs politiques des hommes au pouvoir et les stratégies économiques du marchand.

\section{Missions en Russie : 1781-1782}

Pour préciser le projet commercial, Antoine Anthoine fut envoyé en mission de prospection en Russie en 1781. Au vrai, cette mission se situait au confluent du commercial et du diplomatique. En effet, Antoine Anthoine disposait pour la mener à bien, de lettres de recommandation des ambassadeurs russe et français. Il mit à profit une première étape à Kherson, en 1781, pour jeter les bases d'un futur établissement et rassembler des informations sur les produits disponibles sur place (bois, grains, cuirs, suif, beurre, cire, viande

12. Sur le rôle joué par le drogman ou interprète voir PARIS, Robert, op. cit., p. 225.

13. Emmanuelui, François-Xavier, La Crise marseillaise de 1774 et la chute des courtiers. Contribution à l'histoire du commerce du Levant et de la banque, Paris, 1979.

14. VAN REGEMORTER, Jean-Louis, La Russie méridionale, la mer Noire et le commerce international de 1774 à 1861, thèse Paris I, exemplaire dactylographié, 1983, p. 23-75. 
salée, caviar, cordages, chanvre, toiles, tabac, fer...) et dont les zones de production apparaissaient plus proches de Kherson que de Riga. L'expédition de deux navires en direction de Marseille, sous pavillon russe, constitua, en 1781-1782, un premier test. Dans cette entreprise, les bons contacts noués avec le gouverneur de Kherson, le général Ivan Abramovitch Hannibal, fils du « nègre " de Pierre le Grand, facilitèrent nombre de démarches.

Après avoir appelé un négociant français, Fabre, à Kherson, et fait venir un de ses frères à Constantinople, Anthoine prit le chemin de Petersbourg pour y rencontrer le représentant français, le marquis de Vérac, et le prince Potemkine, proche de Catherine II. Les interlocuteurs montrèrent un réel intérêt pour le projet commercial. Là encore, la convergence évidente entre les objectifs économiques et les perspectives politiques - le projet permettant l'enracinement de la Russie dans cette partie fraîchement conquise - ne manquèrent pas de favoriser l'initiative d'Anthoine. Dans ces conditions, Catherine II autorisa l'acheminement par Kherson du bois de mâture, du bois de construction et des céréales.

Fort de ce soutien Anthoine rentra à Kherson, en 1782, pour y créer sans plus attendre son propre établissement. Celui-ci fut aussitôt confié à ses associés, à savoir son frère Louis et le capitaine Sauron, de La Ciotat : le réseau familial et le réseau professionnel figurent à la base de cette création. Qui plus est, le chancelier de l'ambassade russe à Constantinople participait à la commandite et s'il faisait des affaires médiocres sa principale utilité semble avoir été " d'aplanir les choses avec les autorités ", de limiter les coûts de transaction ${ }^{15}$.

\section{Retour en France : 1782-1783}

Après un court voyage en Pologne afin d'obtenir le droit d'exporter des produits polonais par Kherson, Anthoine se rendit à Versailles (1782-1783). Là, il exposa son projet, cartes à l'appui, au maréchal de Castries, ministre de la Marine, à d'Ormesson, contrôleur général des Finances, et au Comte de Vergennes, ministre des Affaires étrangères, anciennement en poste comme ambassadeur de France à Constantinople. Le projet qui retint l'intérêt des premiers responsables fut ensuite présenté au Roi qui le soutint en accordant à son initiateur un certain nombre d'avantages pour en faciliter l'exécution : suppression de droits et de taxes, prêts (argent et navires), réduction du temps de quarantaine à effectuer au retour de voyage, privilège pour la levée des marins afin de composer au mieux les équipages. Ces avantages avaient, pour contre-partie, l'approvisionnement rapide de l'arsenal de Toulon en produits stratégiques ${ }^{16}$.

15. VAN Regemorter, Jean-Louis, La Russie méridionale ..., op. cit., p. 383.

16. ButI, Gilbert, "Un arsenal méditerranéen et ses aspects forestiers : Toulon (XVIII ${ }^{\mathrm{e}}$ siècle) ", dans Convol, André (dir.), Forêt et Marine, Actes du Colloque international Forêt et Marine, Paris, septembre 1997, Paris, 1999, p. 471-486. 
De retour à Marseille, après avoir fait une halte à Lyon (produits manufacturés) et à Embrun (obsèques de son père) Anthoine créa, en novembre 1783, sa propre société de commerce. Il s'empressa de choisir les bâtiments destinés aux futures opérations et leur attribua de nouveaux noms aux évidentes références politiques : Catherine II, Chevalier Boulgakov, Général Hannibal et Prince Potemkine, à bord desquels étaient exigées des prières quotidiennes pour Catherine II.

Complétant son dispositif commercial, il installa à Kherson une commandite avec pour raison sociale Anthoine frères, Sauron et Compagnie, dans laquelle son frère Félix rejoignait Louis et le capitaine ciotaden Sauron. Antoine Anthoine pouvait également savoir compter, pour y avoir des intérêts, sur la société $J$.V.Gautier. Quelques maisons étrangères imitèrent ces initiatives dans la mesure où d'autres ports de la Méditerranée, comme Gênes, Livourne et Trieste, entreprirent de commercer avec la mer Noire sous pavillon russe.

En guise d'ultimes préparatifs Antoine Anthoine se rendit en Lituanie polonaise, en 1784, avec un maître-mâteur pour sélectionner des arbres sur pied après avoir obtenu l'autorisation du pouvoir politique, en l'occurrence celui du prince Stanislas Poniatowski, neveu du roi de Pologne.

Entre 1784 à 1787, près de 50 bâtiments arrivèrent à Marseille en provenance de la mer Noire dont la moitié pour le compte d'Anthoine; parmi les autres intéressés à ce nouveau circuit figurent de grands noms du négoce marseillais (veuve Councler et fils aîné, Sollicoffre, J.-J. Kick, Rolland frères) ${ }^{17}$.

Les responsables de l'arsenal de Toulon accueillirent, en présence de l'abbé Raynal, les navires d'Anthoine chargés de bois. Cependant, les résultats se révélèrent relativement décevants. Certes le coût était $20 \%$ moins cher que par Riga (37 selon Anthoine, mais 12 \% seulement selon l'intendant Malouet) et les bois, bien que prêts à être embarqués six mois après la coupe contre dix-huit à vingt-quatre pour la Baltique, ne répondaient guère aux exigences de la marine : le ministre de Castries parla même de " rebut des négociants du Nord ${ }^{18}$ ".

Contrairement à l'arsenal, pour lequel les bénéfices se révélèrent illusoires tant la cargaison était médiocre, l'entrepreneur réalisa de "beaux profits " mais dans ses spéculations sur les blés. L'utilisation de la voie politique lui a permis assurément de réduire les " coûts de transaction " pour effectuer une opération personnelle. De l'aveu même d'Anthoine : « l'objet des bois n'était qu'un accessoire dont il avait bien voulu se charger, mais qui ne devait déranger en rien ses opérations personnelles ${ }^{19}$ " Ainsi que le

17. Arch. dép. des Bouches-du-Rhône. Intendance sanitaire, 200 E 540-543.

18. VAN REGEMORTER, Jean-Louis, « Légende ou réalité : Antoine Anthoine, pionnier du commerce marseillais en mer Noire ", dans Hommes, idées, journaux. Mélanges en l'honneur de Pierre Guiral, Paris, 1988, p. 319-325.

19. Mémoire du Sieur Braud à Fabry, directeur de l'arsenal, 27 mai 1784, cité par VAN REGEMORTER, Jean Louis, art. cit., p. 322. 
Produits du Nord et route maritime " ouverte" par Antoine Anthoine en direction de Marseille et Toulon (1784-1787)

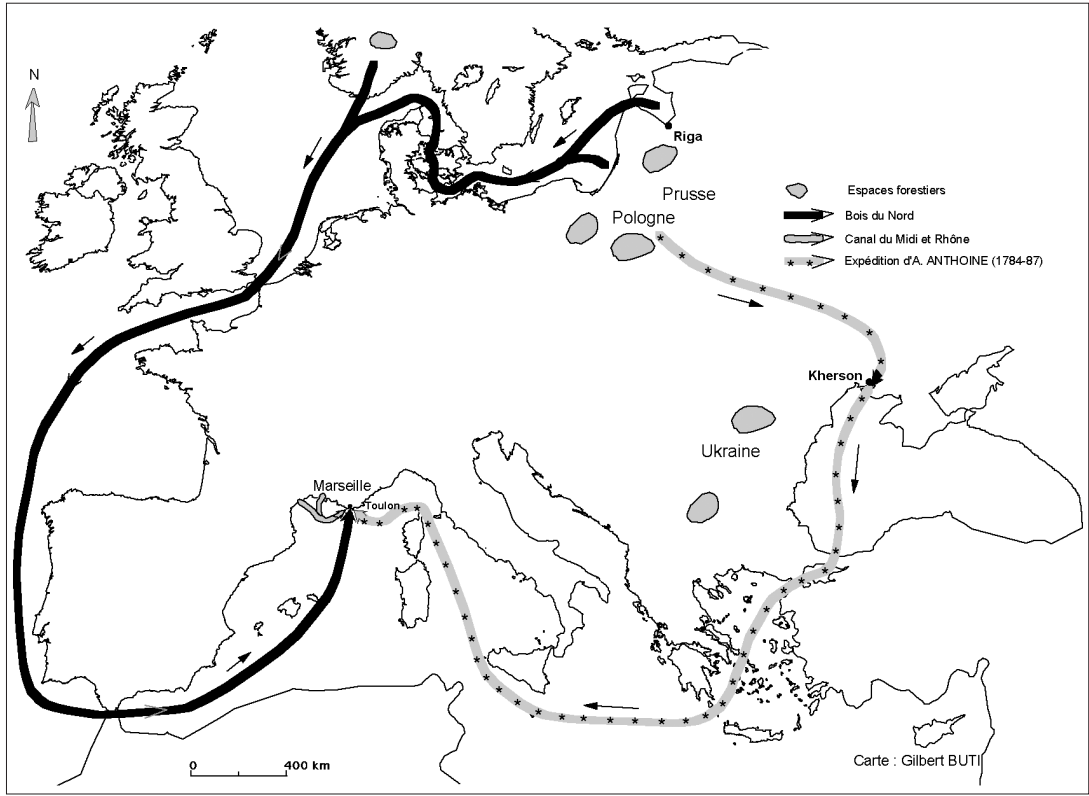

notait amèrement et en toute lucidité Malouet : " Il semble véritablement qu'il ait réservé pour son propre compte l'intelligence et les lumières que je lui connais ${ }^{20}$. " Au vrai, l'échec de son expédition des mâtures en direction de Toulon résultait largement de sa méconnaissance des réseaux traditionnels qui liaient, depuis de longues années, les propriétaires de forêts aux marchands de Riga.

Toutefois, pour renforcer la présence de sa société en Russie méridionale, Anthoine invita ses deux derniers frères, à savoir Charles et Calixte, à se rendre en Ukraine pour y créer un établissement mais ils furent rapidement emportés l'un et l'autre par une épidémie. La reprise de la guerre russo-turque interrompit les relations commerciales en 1787. Antoine Anthoine adopta alors, comme tout " parfait négociant ", une stratégie de repli. De 1787 à 1792, il se tourna vers la Louisiane et les Antilles, SaintDomingue surtout, là " où se levait le soleil des affaires ${ }^{21}$ ", et créa au Cap français un établissement qu'il confia à son frère Félix rentré de Kherson. Cependant, dès la nouvelle de la paix entre la Russie et l'Empire ottoman (1792), son frère Louis, revenu à Marseille, reprit la route de Kherson.

20. Lettre adressée en juillet 1787 par Malouet à Castries, citée par VAN REGEMORTER, Jean-Louis, art. cit., p. 322.

21. CARRIERE, Charles, Richesse du passé marseillais. Le port mondial au XVIII siècle, Marseille, 1979, p. 35. 
En mobilisant sa proche parenté dans cette phase de grande activité, Anthoine a agi comme la plupart des maisons marseillaises pour lesquelles le cadre familial restait le socle des affaires. Cependant, il a su également dans ses activités utiliser avec lucidité les pouvoirs politiques locaux, nationaux et internationaux, soutien d'autant plus important qu'il y avait convergence d'intérêt entre les impératifs politiques et les stratégies entrepreneuriales.

Néanmoins, si le négociant fréquentait les hommes du pouvoir il demeurait encore dans son comptoir ne laissant pas deviner de plans visant à les rejoindre. Les objectifs économiques ne sauraient se réduire à la recherche systématique du pouvoir politique. Néanmoins, les choses paraissent changer, ou ne plus être dissimulées, pour Anthoine une fois fortune assurée. Fortune assurée mais fortune à consolider.

\section{Du comptoir à la toge (1786-1813)}

À la veille de la Révolution, Antoine Anthoine, par sa fortune, sa réputation et la confiance que d'aucuns recherchaient et que d'autres lui accordaient, figurait parmi les négociants qui comptaient sur la place. Toutefois, la consécration passait par l'intégration au groupe dirigeant, synonyme d'honneurs et de prestige. Cette consolidation emprunta trois voies complémentaires : l'accession à la noblesse, le mariage et la " toge municipale ".

\section{La noblesse royale (1786) puis impériale (1808)}

En septembre 1786, sur proposition du maréchal de Castries et du comte de Saint-Priest, Antoine Anthoine fut anobli par Louis XVI ${ }^{22}$. Ses « parrains " politiques étaient bien placés pour connaître et faire reconnaître son action diplomatique et commerciale. De Castries n'avait-il pas suggéré, dès 1783 , qu'une marque de distinction lui soit accordée?

" Les efforts que le Sr Anthoine a faits pendant sa résidence au Levant pour donner de l'extension au commerce [...] son empressement à entrer dans les vues du Gouvernement et à en faciliter le succès par les mémoires instructifs qu'il vous a fournis sur divers articles de nos importations, le sacrifice que ce négociant a fait de quelques années de sa régie à Constantinople $[\ldots]$ tous ces motifs, réunis à votre suffrage que le $\mathrm{Sr}$ Anthoine a su mériter dans tous les temps, sollicitent pour lui des bontés du Roi une marque de distinction ${ }^{23}$."

Au reste, Anthoine atteignit le titre d'écuyer sans passer par l'achat de la charge de Conseiller Secrétaire du Roi comme le faisaient alors la plupart des négociants marseillais ${ }^{24}$. Le titre "traversa la Révolution " puis-

22. Arch. dép. des Bouches-du-Rhône, B 140. Lettres de noblesse en faveur de Sieur Antoine Ignace Anthoine négociant à Marseille, septembre 1786.

23. Arch. nat., $\mathrm{AE} \mathrm{B}^{1}-28, \mathrm{f}^{\circ}$ 149. Lettre de Castries à Saint-Priest, Versailles, 13 mai 1783.

24. CARRIERE, Charles, Négociants marseillais..., op. cit., p. 275-296. 
qu'en septembre 1808, Napoléon Bonaparte, après avoir institué la noblesse impériale, signa des lettres patentes faisant de lui baron de Saint-Joseph, en référence à une propriété acquise, en 1800, dans le terroir de Marseille. Cette bastide paraissait doublement " destinée " à Anthoine : construite en 1740 pour le marquis Louis Sauveur de Villeneuve, ambassadeur de France à Constantinople, elle avait été vendue trente ans plus tard au négociant dauphinois Joseph Hugues, la plus grosse fortune de Marseille à la veille de la révolution.

La confirmation de la noblesse par Napoléon n'était pas tout à fait étrangère aux liens familiaux qui unissaient alors les deux hommes.

\section{Le mariage et la nouvelle sphère familiale (1786)}

En janvier 1786, peu avant son anoblissement, Antoine Anthoine avait épousé Marie Anne Rose Clary et s'était ainsi glissé dans le groupe restreint des dirigeants de la cité, parmi ceux qui tiennent le port et qui dirigent la ville étant donné l'accaparement du pouvoir municipal par les acteurs économiques. Née en 1764, Rose Clary était la fille de François, négociant, premier échevin (1764) et député du Commerce, et avait pour parrain Nicolas Samatan (négociant et échevin), et pour marraine Anne Marie Olive, femme de négociant et de premier échevin. Une de ses sœurs, Julie, épousa Joseph Bonaparte et une autre, Désirée, fiancée en 1795 à Napoléon, se maria finalement avec Bernadotte ${ }^{25}$.

Les témoins du mariage d'Antoine Anthoine, comme les parrains et marraines des quatre enfants qui naquirent de cette union, appartenaient également au monde des affaires et de la magistrature, du comptoir et de la toge. Par la parenté nouvelle et les réseaux ainsi ouverts, le mariage a constitué pour Anthoine, dont la stratégie n'était en rien originale, un acte essentiel pour pénétrer dans le cercle étroit des notables de la place.

\section{La Mairie (1790-1805)}

Bien qu'Anthoine se trouvât parmi les treize conseillers municipaux de 1790, le cheminement fut tortueux et relativement long pour accéder aux responsabilités édilitaires en cette période révolutionnaire. Les activités économiques et les prétentions politiques rencontrèrent la guerre, le blocus et la paralysie du port de Marseille, en 1793, ainsi que la Terreur, avec l'exécution, en 1793-1794, de grands négociants à commencer par les Seimandy, ses premiers protecteurs.

Anthoine s'installa alors à Gênes avec plusieurs membres de sa bellefamille - Joseph Bonaparte, Désirée et Nicolas Clary - qui reçurent la visite de Napoléon en juillet 1794. Toutefois, Anthoine put regagner Marseille à la suite de contacts pris par les représentants en mission pour obtenir,

25. DIOQUE, Georges, op. cit., p. 131-132. 
en cette période de pénurie et de vie chère, l'envoi " d'autant de farine et de blé qu'il pourra en réunir ". La " politique annonaire " ne se trouvaitelle pas, par définition même, à la croisée des chemins du politique et de l'économique ${ }^{26}$ ? En 1795, il fut nommé membre du "Bureau des subsistances " (ex-Bureau d'Abondance) chargé du ravitaillement de la cité, alors que Bonaparte lui proposait de conduire une mission militaire à Constantinople.

Les portes du pouvoir demeurèrent néanmoins difficiles à franchir. En 1803, Anthoine échoua dans sa tentative pour entrer au Conseil général du commerce, le choix se portant sur Dominique Audibert soutenu par la puissante Chambre de commerce. Il connut un nouvel échec l'année suivante en voulant entrer cette fois au Corps législatif, étant pourtant soutenu par les Hautes-Alpes originelles et les Bouches-du-Rhône adoptives ${ }^{27}$.

Finalement, Napoléon Bonaparte qui souhaitait disposer d'un négociant fidèle à la tête de la ville de Marseille le nomma maire le 30 juillet $1805^{28}$. Il fut " intronisé " dans ses fonctions à la Loge des marchands, salle de la Bourse, espace des stratégies négociantes et cœur du pouvoir de la cité ${ }^{29}$. Le baron de Saint-Joseph resta maire de Marseille jusqu'à sa démission, pour raison de santé, en 1813.

\section{La toge au service du comptoir (1805-1813)?}

Les réseaux marchands et familiaux, les stratégies entrepreneuriales et les services rendus aux responsables politiques ont permis à Anthoine d'accéder au pouvoir local et d'approcher les pouvoirs nationaux et internationaux. Un jeu de réciprocité se dessine entre ces pouvoirs et les affaires. Les nouvelles responsabilités municipales ont-elles été recherchées puis actionnées par Anthoine pour nourrir des visées commerciales? Il demeure délicat de le savoir. Les acteurs économiques chercheraient-ils systématiquement à s'emparer de pouvoirs politiques pour les mettre au service de leurs ambitions marchandes? Les initiatives prises par le maire de Marseille s'inscriraient-elles dans cette perspective?

Le combat mené pour le rétablissement de la franchise du port constitue un premier test qui dépasse, il est vrai, les seules affaires du maire de la ville. L'édit d'affranchissement du port instauré sous Colbert (1669) avait été révoqué par la Convention en décembre 1794. Or, pour Anthoine

26. BuTI, Gilbert, « La traite des blés et la construction de l'espace portuaire de Marseille (XVII ${ }^{\mathrm{e}}$-XVIII ${ }^{\mathrm{e}}$ siècle) ", dans MARIN, Brigitte, VIRLOUVET, Catherine (dir.), Nourrir les cités de Méditerranée, Antiquité - Temps modernes, Paris, 2003, p. 769-799.

27. Dioque, Georges, op. cit., p. 143-144.

28. Comme la moitié des négociants marseillais, Antoine Anthoine était impliqué dans la franc-maçonnerie, étant garde des Sceaux de la mère loge de saint Jean d'Écosse en 1808. Ce réseau eut sans nul doute une influence dans son itinéraire sans que nous puissions à la lumière des travaux actuels en déterminer la part.

29. GIRAUD, Pierre, Marseille. L'Hôtel de ville, la Loge. Les deux Maisons du roi, Marseille, 1962. 
comme pour beaucoup de négociants marseillais, le rétablissement de la franchise semblait indispensable pour assurer le renouveau commercial de Marseille. Cependant, malgré les doléances appuyées du baron de SaintJoseph, Napoléon se montra intraitable et refusa la requête : "Les Marseillais ne peuvent être à la fois étrangers et Français ${ }^{30}$."

En 1805, année de sa nomination à la mairie de Marseille, Antoine Anthoine publia un ouvrage appelé à un réel succès : Essai historique sur le commerce et la navigation de la mer Noire ${ }^{31}$. Rédigé en 1804, ce texte, à la fois ouvrage-souvenir et manuel de commerce, n'est peut-être pas étranger au choix de Napoléon pour l'attribution de la " toge municipale ". Il constituait d'abord un rappel élogieux de son action passée de " pionnier de la mer Noire ", une marche triomphale vers ces nouveaux espaces masquant les déboires de la Marine à propos des bois de mâture ${ }^{32}$. En imbriquant des calculs économiques et des arrière-pensées politiques, l'Essai se présentait également comme une invitation à intensifier les échanges avec la mer Noire et comme une étude de marché (monnaies, coût du fret, taxes, prix, usages, plans, conseils...). Il reste difficile de mesurer le rôle joué par cette publication dans l'impulsion donnée aux échanges avec les territoires riverains de la mer Noire, échanges qui ne prirent une réelle ampleur que dans les décennies suivantes.

Toutefois, en 1820, après avoir renoncé à la mairie et pris quelque distance avec le grand négoce, Antoine Anthoine, âgé alors 71 ans, proposa une réédition de son texte étant donné " l'accroissement prodigieux du commerce et de la navigation " dans un nouveau cadre politique. Dans cette réédition, qui se voulait immédiatement utilisable par les milieux d'affaires, on trouvait une description détaillée des villes marchandes (Kherson, Odessa...) et un intérêt pour la liaison entre la mer Noire et la mer Caspienne, renouant ainsi avec de vieux rêves de jeunesse, ceux du temps de sa nomination à Constantinople.

Au vrai, Antoine Anthoine demeurait attentif aux questions de la mer Noire car il avait maintenu des liens commerciaux avec cet espace. Malgré les périodes troublées, la maison créée en 1782 existait toujours lorsqu'il dirigeait la municipalité marseillaise et se trouvait administrée par des proches. Il avait confié, en 1791, à un cousin d'Embrun, Antoine Rous de La Mazelière, son établissement de Constantinople dans lequel il avait fait entrer, en 1803, en qualité de commis Bruno-Xavier Rostand, fils du maîtredrapier qui l'avait accueilli, vers 1767, à Marseille et l'avait mis en relation avec les Seimandy. Enfin, à Kherson était affecté Albrand, proche collaborateur d'Antoine Anthoine alors que la ville avait pour gouverneur le comte

30. La franchise portuaire fut rétablie par le Comte d'Artois, en 1814, et suivie par la suppression de la taxe de $20 \%$ ad valorem en 1815. La franchise fut supprimée définitivement en 1817.

31. AnTHoIne, Antoine, Essai historique sur le commerce et la navigation de la mer Noire, Paris, 1805.

32. VAN REgEMORTER, Jean Louis, art. cit., p. 319. 
de Saint-Priest, fils de l'ancien ambassadeur de France à Constantinople. Ainsi s'esquissait un réseau de seconde génération.

Dans cette dernière phase apparaissent plus nettement les connexions et les logiques qui existent entre économie et pouvoir, avec comme soubassement une puissante parentèle et de solides fidélités. Fidélité réciproque quand on songe qu'en 1826, lors de l'enterrement d'Antoine Anthoine au cimetière Saint Charles, à Marseille, c'est Alexis Rostand, frère de Bruno-Xavier et futur maire de Marseille (1830), qui prononça un des discours funèbres.

Cette étude menée " au ras du sol " présente nécessairement un certain nombre de " chaînons manquants "; elle ne nous permet pas d'atteindre, dans toute son épaisseur, la complexité des liens tissés entre le monde de la marchandise et celui des pouvoirs à quelque degré que ce soit. En cela elle ne se veut en rien exemplaire et n'a pas la prétention d'être un modèle. Qui plus est ce destin, à plus d'un titre extraordinaire, ne semble guère opératoire à qui souhaite faire émerger des constantes et dégager des logiques de fonctionnement généralisables. Toutefois, ne souligne-t-il pas la place, la fonction et le rôle des individus, des familles et des groupes à l'intérieur de stratégies d'affaires et de pouvoir?

Le passage du comptoir à la toge ne signifie pas renoncement à une activité mais ouverture de celle-ci par la mobilisation des compétences et des talents au sein d'une fratrie, d'une famille ou d'un front de parenté. Loin de l'éloigner des pratiques marchandes, les "missions diplomatiques " et la carrière politique amorcée par Anthoine lui permettrent d'ouvrir le champ d'action des réseaux économiques au monde politique.

D'autres trajectoires devraient enrichir un dossier que Charles Carrière avait ouvert de manière globale dans son étude consacrée aux négociants marseillais. D'ailleurs, afin de dresser le portrait du Parfait négociant ne se proposait-il pas de brosser le parcours de quelques acteurs économiques, éclatants ou non? Son dernier ouvrage - ouvrage posthume - consacré à une grande figure de la place, à savoir Georges Roux de Corse, s'inscrit dans cette démarche visant à décrypter les relations qui existent entre économie et pouvoir ${ }^{33}$. Toutefois, comme Georges Roux et Antoine Anthoine, d'autres figures pourraient prendre place dans cette galerie de portraits - Jacques Seimandy, Hugues l'aîné, Dominique Audibert et bien d'autres afin de dégager les connexions établies entre les pratiques marchandes et les pouvoirs. Les liens avec "l'internationale huguenote" ou les réseaux languedociens ne sauraient tout expliquer.

Au reste, une exploration menée également sur de plus modestes places conduirait à saisir le subtil jeu de réciprocité qui existe entre les différents pouvoirs et les activités économiques. Les réseaux familiaux des élites, les

33. CARriere, Charles, Goury, Michel, Georges Roux de Corse. L'étrange destin d'un armateur marseillais (1703-1792), Marseille, 1990. 
plus aisés sans doute à reconstituer, dévoilent déjà le jeu complexe établi à partir des affaires maritimes avec les représentants de la robe et de l'épée, de la toge et du comptoir. Ils font apparaître des glissements, selon des stratégies individuelles ou des fronts de parenté, entre le pont du navire, l'échoppe ou la boutique et diverses magistratures. À partir de ce brassage et en prenant appui sur des analyses conduites sur la longue durée, certains aspects pressentis à grands traits dans les ports secondaires, du XVI ${ }^{\mathrm{e}}$ au XIX ${ }^{\mathrm{e}}$ siècle, tout comme à Marseille malgré l'insuffisance des études pourraient être validés. Ainsi en est-il du rôle de la parenté directe, du poids des stratégies familiales dans lesquelles les femmes jouent un rôle essentiel dans la circulation des biens, matériels ou non, et de la mise en place de nouvelles configurations autour de pouvoirs émergents. 


\section{RESUME}

Descendant d'une famille de petits notables du Dauphiné, Antoine Anthoine (1749-1826) qui n'a pas pu accéder à la magistrature se destine à la marchandise. Après un apprentissage à Marseille au comptoir des Seimandy, il est nommé, par ces négociants, régisseur pour leur commandite de Constantinople.

La mobilisation de sa proche parenté et les soutiens des pouvoirs politiques locaux, nationaux et internationaux lui permettent d'obtenir une mission destinée à ouvrir le commerce de la Russie méridionale à la France. La convergence d'intérêt entre les impératifs politiques et les stratégies marchandes favorisent plusieurs expéditions en direction de Marseille (blé) et de Toulon (bois), à travers la mer Noire et non plus par la Baltique (1784-1787)

Si le bilan financier des opérations appelle quelques réserves, Anthoine apparaît, à la veille de la Révolution, comme un des plus importants négociants de Marseille. L'intégration au groupe dirigeant passe alors par trois voies complémentaires : l'accession à la noblesse (1786), le mariage (en 1786, avec Rose Clary, fille de négociant et échevin de la ville, et par-là beau-frère de Joseph Bonaparte et de Bernadotte) et la " toge municipale " (1805-1813) pour ce baron d'Empire (1808) qui n'a de cesse de se présenter comme le " pionnier du commerce marseillais en mer Noire. "

Cet itinéraire montre les connexions établies entre pratiques marchandes et pouvoirs. Il ne se présente pas comme un modèle et n'est guère opératoire pour qui souhaiterait faire émerger des constantes ou dégager des logiques de fonctionnement. Cette trajectoire souligne la place, la fonction et le rôle des individus, des familles et des groupes à l'intérieur de stratégies d'affaires et de pouvoir. Le passage du comptoir à la toge ne signifie pas renoncement à une activité mais ouverture de celle-ci par la mobilisation des compétences et des talents au sein d'une fratrie, d'une famille ou d'un front de parenté.

\section{ABSTRACT}

Descendant of a family of small notables of Dauphine, Antoine Anthoine (17491826) was not able to reach the judiciary. He goes to the goods and after a apprenticeship in Marseilles at the counter of Seimandy, he is appointed, by these traders, manager for their company of Constantinople.

The mobilization of its close relationship and the supports of the local, national and international political powers, allow him to obtain a mission intended to open the trade of Southern Russia in France. The convergence of interest between the political requirements and the commercial strategies favour several expeditions in the direction of Marseilles (wheat) and of Toulon (naval stores), through the Black Sea and either by the Baltic (1784-1787).

If the financial balance of the operations calls some reserves, Anthoine appears, on the eve of the Revolution, as one of the most important traders of Marseilles. The integration to the steering group passes then by three complementary ways : the entry in the nobility (1786), the marriage (in 1786, with Rose Clary, girl of trader and alderman of the city, and by there brother-in-law of Joseph Bonaparte and Bernadotte) and the "municipal toga" (1805-1813) for this baron of Empire (1808) who has respite to appear as the "pioneer of the trade of Marseilles in Black Sea".

This destiny shows the connections established between commercial practices and powers. It does not appear as a model and is not hardly operating to whom would like to bring to the foreground constants or logics of functioning. This trajectory underlines the place, the function and the role of the individuals, the families and the groups inside strategies of business and power. The passage of the counter in the toga does not mean renunciation of an activity but an opening of this one by the mobilization of the competence and the talents within a family or within a relationship. 
